Supporting Information

\title{
Biological Sample-Compatible Ratiometric Fluorescent Molecularly Imprinted Polymer Microspheres by RAFT Coupling Chemistry
}

Yuxia Hou, Yiwei Zou, Yan Zhou, and Huiqi Zhang*

State Key Laboratory of Medicinal Chemical Biology, Key Laboratory of Functional Polymer Materials (Ministry of Education), Collaborative Innovation Center of Chemical Science and Engineering (Tianjin), and College of Chemistry, Nankai University, Tianjin 300071, P. R.

China.E-mail: zhanghuiqi@nankai.edu.cn<smiles>CC(CC(C)(Cc1ccccc1)c1ccccc1)C(C)(C)c1ccccc1</smiles>

CDB<smiles>CC(C)NC(=O)CCC(C)(C)CCC(=S)SC(=S)c1ccccc1</smiles>

PNIPAAm macro-CTA: $M_{\mathrm{n}, \mathrm{NMR}}=7840$

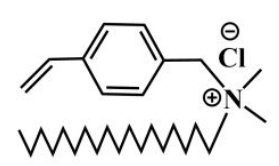

OVDAC

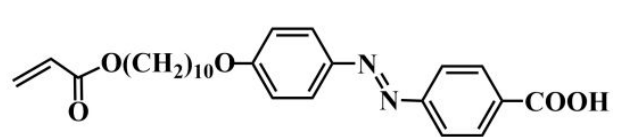

A-Azo

Scheme S1. Chemical structures of some reagents used in the study.

Synthesis and characterization of a well-defined poly(NIPAAm) (PNIPAAm) with a dithioester end-group (i.e., PNIPAAm macro-CTA, Scheme S1)

The well-defined PNIPAAm macro-CTA was synthesized via the RAFT polymerization of NIPAAm following a literature method (F. Ganachaud, et al., Macromolecules 2000, 33, 6738) as follows: NIPAAm (37.50 mmol), AIBN (0.056 mmol), CDB (0.28 mmol), and dioxane (15 $\mathrm{mL})$ were added into a one-neck eggplant-shaped flask $(25 \mathrm{~mL})$ successively. The reaction mixture was degassed with five freeze-pump-thaw cycles, sealed, and then placed in a thermostatted oil bath at $60{ }^{\circ} \mathrm{C}$. After being stirred for $48 \mathrm{~h}$, the reaction solution was diluted with tetrahydrofuran (THF) $(10 \mathrm{~mL})$, and then precipitated into pentane twice to obtain a light pink polymer, which was dried under vacuum at room temperature for $48 \mathrm{~h}$ to provide the desired product (yield: 59\%).

Table S1 presents the molecular weight $\left(M_{\mathrm{n}, \mathrm{GPC}}\right)$ and molar-mass dispersity $(\nexists)$ of the resulting PNIPAAm macro-CTA, which were determined by GPC on a Waters system equipped with a set of three Ultrastyragel columns (HT2, HT3, and HT4; $30 \mathrm{~cm} \times 7.8 \mathrm{~mm} ; 10$ 
$\mu \mathrm{m}$ particles; exclusion limits: 100-10000, 500-30000, and 5000-600000 g/mol, respectively), a Waters 515 HPLC pump, a Waters 717plus autosampler, and an online Waters 2414 refractive index detector maintained at $35^{\circ} \mathrm{C}$. THF was used as the mobile phase $(1 \mathrm{~mL} / \mathrm{min})$, and polystyrene samples were used as the standards.

Table S1. Molecular weight and molar-mass dispersity data of PNIPAAm macro-CTA.

\begin{tabular}{c|c|c|c}
\hline Sample & $M_{\mathrm{n}, \mathrm{GPC}}{ }^{a}$ & $\bigoplus^{a}$ & $M_{\mathrm{n}, \mathrm{NMR}}{ }^{b}$ \\
\hline PNIPAAm macro-CTA & 8340 & 1.28 & 7840 \\
\hline
\end{tabular}

${ }^{a} M_{\mathrm{n}, \mathrm{GPC}}$ and $Ð$ are the number-average molecular weight and molar-mass dispersity of PNIPAAm macro-CTA determined by GPC. ${ }^{b} M_{\mathrm{n}, \mathrm{NMR}}$ is the number-average molecular weight of PNIPAAm macro-CTA determined by ${ }^{1} \mathrm{H}$ NMR.

The absolute molecular weight of PNIPAAm macro-CTA was measured by ${ }^{1} \mathrm{H}$ NMR (Varian Unity plus-400 spectrometer, $400 \mathrm{MHz}$ ). Figure S1 presents the ${ }^{1} \mathrm{H}$ NMR spectrum of PNIPAAm macro-CTA, from which the number-average molecular weight of the polymer $\left(M_{\mathrm{n}, \mathrm{NMR}}\right)$ was derived to be $7840 \mathrm{~g} / \mathrm{mol}$ by using the following formula (Table $\mathrm{S} 1$ ):

$$
M_{\mathrm{n}, \mathrm{NMR}}=10 \times\left(S_{\mathrm{c}} / S_{\mathrm{f}}\right) \times M_{\mathrm{NIPAAm}}+M_{\mathrm{CDB}}
$$

where $S_{\mathrm{c}}$ and $S_{\mathrm{f}}$ refer to the integral of the peak $c$ around $3.85 \mathrm{ppm}$ and that of the peaks $f$ between 7.2 and $8.2 \mathrm{ppm}$, respectively, $M_{\text {NIPAAm }}$ the molar mass of NIPAAm, and $M_{\mathrm{CDB}}$ the molar mass of CDB.

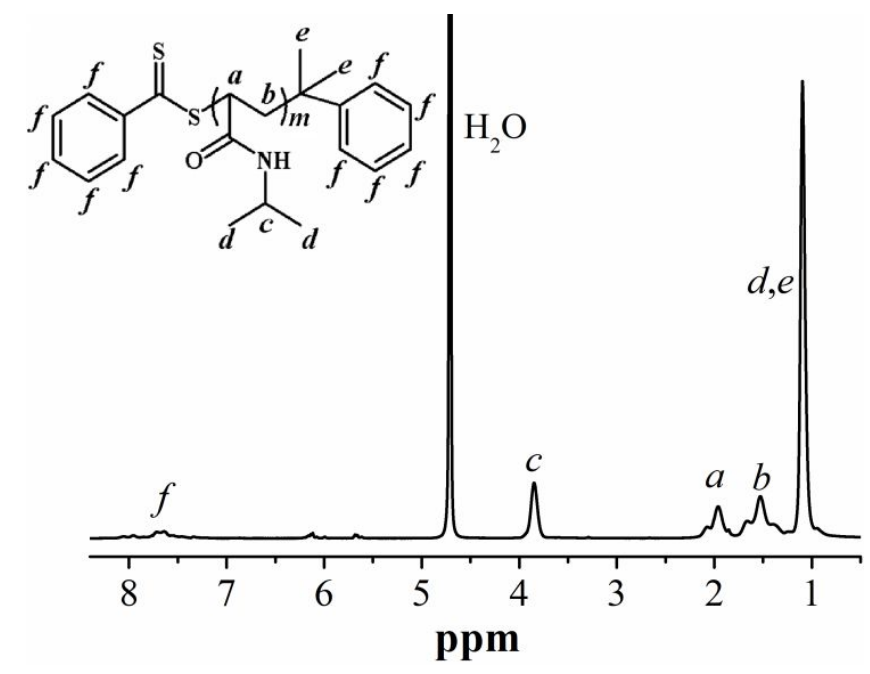

Figure S1. ${ }^{1} \mathrm{H}$ NMR spectrum of PNIPAAm macro-CTA in $\mathrm{D}_{2} \mathrm{O}$. 


\section{Preparation of a solution of red CdTe QDs with surface-bound OVDAC in methanol}

To a one-neck round-bottom flask $(500 \mathrm{~mL})$ were added an aqueous solution of red CdTe QDs (which was prepared following a literature method (S. Wang, et al., Anal. Chem. 2017, 89, 5646)) and OVDAC (which was prepared according to the reported method (W. Zhang, et al., Angew. Chem., Int. Ed. 2014, 53, 12489)) (540 mg). After the above mixture was stirred at room temperature for $2 \mathrm{~h}$, it was extracted with chloroform $(270 \mathrm{~mL})$. The solvent of the chloroform phase was then removed by using a rotovaporator, and the resulting solid was dissolved into methanol $(270 \mathrm{~mL})$. The above-obtained solution of red CdTe QDs with surface-bound OVDAC in methanol was directly used for the synthesis of core-shell-structured red CdTe QD-labeled polymer particles (entry 2, Table 1).

Preparation of "living" poly(MAA-co-EGDMA) microspheres with surface-bound dithioester and vinyl groups (entry 1 in Table 1)

"Living” poly(MAA-co-EGDMA) microspheres with surface-bound dithioester and vinyl groups were synthesized via RAFTPP following the literature procedure (Y. Ma, et al., J. Mater. Chem. B 2019, 7, 2474), but with some modification: To a one-neck round-bottom flask $(100 \mathrm{~mL})$ with a magnetic stir bar inside were added a mixture of dried acetonitrile and methanol (9:1 v/v, $60 \mathrm{~mL})$ and MAA (1.00 mmol). EGDMA (5.00 mmol), CDB (0.22 mmol), and AIBN $(0.11 \mathrm{mmol})$ were then added into the above solution successively. The resulting purple solution was then purged with argon for $30 \mathrm{~min}$, sealed, magnetically stirred (180 rpm) at $60{ }^{\circ} \mathrm{C}$ for $12 \mathrm{~h}$. The formed polymer particles were collected by centrifugation, washed thoroughly with methanol, and then dried at $40{ }^{\circ} \mathrm{C}$ under vacuum to a constant weight, leading to the desired light pink polymer particles (yield: $31 \%$ ) (entry 1 in Table 1).

Preparation of the grafted dual fluorescent DCPAc-MIP/CP particles (i.e., polym@rQD-polym2@NBD-MIP(DCPAc)@PNIPAAm and polym@rQD-polym2@NBD-CP(DCPAc)@PNIPAAm, entries 5,6, Table S2).

Preparation of “living” poly(MAA-co-EGDMA) microspheres with surface-bound dithioester and vinyl groups (i.e., Poly(MAA-co-EGDMA)2, entry 1, Table S2) and core-shell-structured red CdTe QD-labeled polymer particles (i.e., polym@rQD-polym2, entry 2, Table S2) 
Enough amounts of "living" poly(MAA-co-EGDMA) microspheres with surface-bound dithioester and vinyl groups (i.e., Poly(MAA-co-EGDMA)2, entry 1 in Table S2, Figure S2a) and core-shell-structured red CdTe QD-labeled polymer particles (i.e., polym@rQD-polym2, entry 2 in Table S2, Figure S2b) were first prepared following the procedure as for the entries 1 and 2 in Table 1.

Table S2. Synthetic and characterization data of the "living" poly(MAA-co-EGDMA) particles, red CdTe-labeled core-shell polymer particles, and ungrafted/grafted dual fluorescent DCPAc-MIP/CP particles.

\begin{tabular}{clcccc}
\hline Entry & \multicolumn{1}{c}{ Sample } & $\begin{array}{c}\Delta W \\
(\%)^{a}\end{array}$ & $\begin{array}{c}D_{\mathrm{n}, \mathrm{SEM}} \\
(\mu \mathrm{m})^{b}\end{array}$ & $\begin{array}{c}U^{b} \\
\text { Contact } \\
\text { angle }()^{c}\end{array}$ \\
\hline 1 & Poly(MAA-co-EGDMA)2 & - & 1.074 & 1.004 & $126.9 \pm 2.8$ \\
2 & Polym@rQD-polym2 & 76.1 & 1.288 & 1.011 & $119.1 \pm 1.9$ \\
3 & Polym@rQD-polym2@NBD-MIP(DCPAc) & 21.9 & 1.392 & 1.005 & $124.8 \pm 1.5$ \\
4 & Polym@rQD-polym2@NBD-CP(DCPAc) & 22.7 & 1.403 & 1.006 & $125.5 \pm 0.7$ \\
5 & Polym@rQD-polym2@NBD-MIP(DCPAc)@PNIPAAm & 8.8 & 1.410 & 1.006 & $71.4 \pm 1.5$ \\
6 & Polym@rQD-polym2@NBD-CP(DCPAc)@PNIPAAm & 7.9 & 1.418 & 1.007 & $70.4 \pm 1.1$ \\
\hline
\end{tabular}

${ }^{a}$ Increased weight percentage of the polymer particles after each surface modification step in comparison with the starting polymer particles; ${ }^{b} D_{\mathrm{n}, \mathrm{SEM}}$ and $U$ refer to the number-average diameter and size distribution index of the particles determined by SEM, respectively; ${ }^{c}$ The static water contact angles of the polymer films.
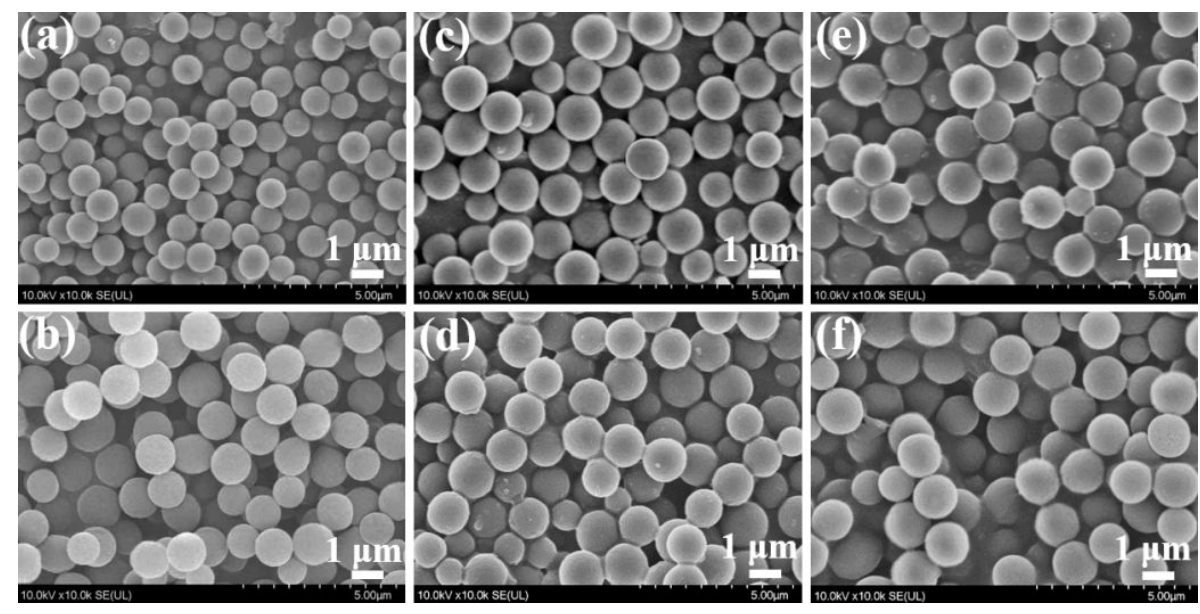

Figure S2. SEM images of poly(MAA-co-EGDMA)2 (a), polym@rQD-polym2 (b), polym@rQD-polym2@NBD-MIP(DCPAc) (c), polym@rQD-polym2@NBD-CP(DCPAc) (d), polym@rQD-polym2@NBD-MIP(DCPAc)@PNIPAAm (e), and polym@rQD-polym2@NBD-CP(DCPAc)@PNIPAAm (f). 
Preparation of the ungrafted dual fluorescent DCPAc-MIP/CP particles (i.e., polym@rQD-polym2@NBD-MIP(DCPAc) and polym@rQD-polym2@NBD-CP(DCPAc), entries 3,4, Table S2)

The ungrafted dual fluorescent DCPAc-MIP microspheres (entry 3 in Table S2, Figure S2c) were prepared following the same procedure as for the ungrafted dual fluorescent 2,4-D-MIP microspheres (entry 3 in Table 1) except that DCPAc was used to replace 2,4-D in the third synthetic step in Scheme 1a (i.e., surface imprinting step).

The ungrafted dual fluorescent DCPAc-CP particles were also prepared and purified under the identical conditions except that DCPAc was omitted (entry 4 in Table S2, Figure S2d).

Preparation of the grafted dual fluorescent DCPAc-MIP/CP particles (i.e., polym@rQD-polym2@NBD-MIP(DCPAc)@PNIPAAm and polym@rQD-polym2@NBD-CP(DCPAc)@PNIPAAm, entries 5,6, Table S2)

The grafted dual fluorescent DCPAc-MIP/CP particles (i.e., dual fluorescent DCPAc-MIP/CP particles with surface-grafted PNIPAAm brushes) (entries 5,6 in Table S2, Figure S2e,f) were prepared via the simple coupling reaction of PNIPAAm macro-CTA (Scheme S1) with vinyl groups on the ungrafted dual fluorescent DCPAc-MIP/CP particles in the presence of a free radical initiator following the same procedure as for the grafted dual fluorescent 2,4-D-MIP/CP particles (entries 5,6, Table 1).

Quantification of the amount of dithioetser groups on the "living" poly(MAA-co-EGDMA) microspheres

The quantification of the amount of dithioester groups on "living" poly(MAA-co-EGDMA) microspheres was carried out by first quantitatively transforming the dithioester groups on the "living" poly(MAA-co-EGDMA) particle surfaces into thiol groups through aminolysis reaction and then quantifying the content of the resulting surface thiol groups by using Michael addition click reaction. The detailed procedure is shown below:

Transforming dithioester groups on "living” poly(MAA-co-EGDMA) microspheres into thiol groups by aminolysis 
The dithioester groups on "living" poly(MAA-co-EGDMA) microspheres (entry 1, Table 1, Figure S3a) were transformed into thiol groups through aminolysis following a literature method (T. Zhou, et al., RSC Adv. 2014, 4, 30292): To a one-neck round-bottom flask (10 mL) were added "living" poly(MAA-co-EGDMA) microspheres with surface-bound dithioester groups $(50 \mathrm{mg})$, ethylene diamine $(27.5 \mu \mathrm{L})$, triethylamine $(57.5 \mu \mathrm{L})$, and DMF $(825 \mu \mathrm{L})$ successively. The reaction mixture was purged with argon for 5 minutes and was stirred at 50 ${ }^{\circ} \mathrm{C}$ for $16 \mathrm{~h}$. The $\mathrm{pH}$ value of the particle suspension was then tuned to 5 by using $0.1 \mathrm{M} \mathrm{HCl}$. Finally, the poly(MAA-co-EGDMA) microspheres with surface-bound thiol groups (Figure $\mathrm{S} 3 \mathrm{~b})$ were obtained by washing the resulting particles with water until the $\mathrm{pH}$ of the supernatant became neutral. The successful transformation of dithioester groups into thiol groups was confirmed by the color change of the polymer particles from purple to white (Figure S3a,b).
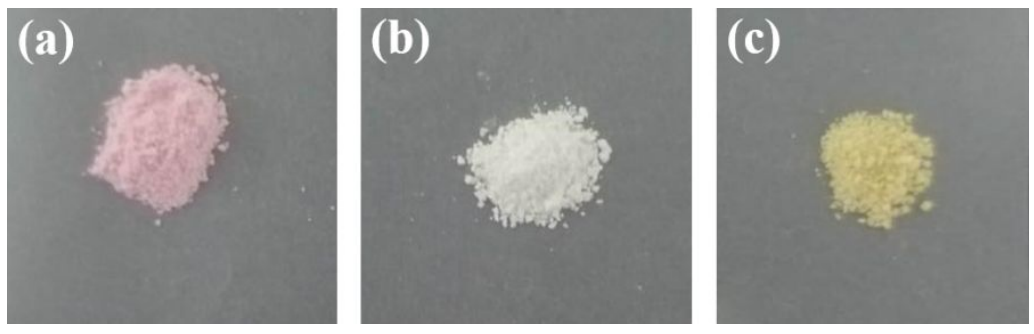

Figure S3. The colors of "living" poly(MAA-co-EGDMA) microspheres with surface-bound dithioester groups (a), poly(MAA-co-EGDMA) microspheres with surface-bound thiol groups (b), and poly(MAA-co-EGDMA) microspheres with surface-bound azobenzene groups (c).

\section{Determination of the content of thiol groups on polymer microspheres}

The content of thiol groups on the above-obtained poly(MAA-co-EGDMA) microspheres was determined through their Michael addition click reaction with an acrylate-type azobenzene compound (A-Azo, Scheme S1) as shown below: To a one-neck round-bottom flask $(10 \mathrm{~mL})$ were added poly(MAA-co-EGDMA) microspheres with surface-bound thiol groups (10 mg) and $\mathrm{DMF} / \mathrm{methanol}(3: 1 \mathrm{v} / \mathrm{v} ; 1 \mathrm{~mL})$, triethylamine $(12.34 \mu \mathrm{mol}, 1.72 \mu \mathrm{L})$, and A-Azo $\left(C_{0}=\right.$ $12.34 \mu \mathrm{mol}, 5.58 \mathrm{mg}$ )(yellow color) successively. The reaction mixture was purged with argon for 5 minutes and then was stirred at $40{ }^{\circ} \mathrm{C}$ for $6 \mathrm{~h}$. Afterwards, the reaction system was centrifuged and the resulting polymer particles were washed with DMF/methanol $(3: 1 \mathrm{v} / \mathrm{v})$ thrice $(3 \times 9 \mathrm{~mL})$ (leading to a pale yellow polymer (Figure S3c)). All the collected supernatants were combined to one mixture, which was diluted with DMF/methanol $(3: 1 \mathrm{v} / \mathrm{v})$ 
to a fixed volume $(50 \mathrm{~mL})$. The content of A-Azo remaining in the continuous phase of the reaction system $(F)$ was then derived through quantifying the concentration of A-Azo in the above-diluted supernatant mixture (which was further diluted 4 times prior to the UV-vis measurement) by comparing the determined UV-vis absorption (at $359 \mathrm{~nm}$ ) of A-Azo in the finally diluted solution with a pre-established calibration curve of A-Azo in DMF/methanol $(3: 1 \mathrm{v} / \mathrm{v})$ (i.e., a linear plot of UV-vis absorption at $359 \mathrm{~nm}$ of A-Azo versus A-Azo concentration). The amount of the reacted A-Azo during the above Michael addition reaction between A-Azo and thiol groups on poly(MAA-co-EGDMA) particles was thus derived to be 144.6 $\mu \mathrm{mol} / \mathrm{g}$ by using the equation $C_{0}-F$, which should be equal to the content of surface-bound thiol groups on poly(MAA-co-EGDMA) particles because of the typical characteristics of the Michael addition click reaction.

Since the dithioester groups on poly(MAA-co-EGDMA) particle surfaces can be quantitatively transformed into thiol groups through aminolysis reaction, the amount of ditioester groups on poly(MAA-co-EGDMA) particles should also be equal to the above-determined thiol content on the polymer particles (i.e., $144.6 \mu \mathrm{mol} / \mathrm{g}$ ).

\section{Quantification of the amount of CdTe QDs on the core-shell-structured red CdTe QD-labeled polymer particles (i.e., polym@rQD-polym, entry 2, Table 1)}

Based on the experimental observation that all CdTe QDs added into the polymerization system during the preparation of polym@rQD-polym particles in the second-step reaction were incorporated into the polymer particles and no CdTe QDs were left in the solvent phase after the surface-initiated RAFT polymerization (the solvent phase shows no fluorescence), we have also quantified the content of CdTe QDs in the second layer of polym@rQD-polym particles (entry 2, Table 1). To this end, we first determined the total amount ( $W=126.2 \mathrm{mg})$ of CdTe QDs used in the second-step reaction (through evaporating the solvent of the QDs solution and weighting the dried QDs). Therefore, the obtained polym@rQD-polymer particles (492.8 $\mathrm{mg}$ ) in the second-step reaction contain $126.2 \mathrm{mg}$ of CdTe QDs. Since polym@rQD-polym particles showed a weight increase percentage of $82.5 \%$ in comparison with the starting "living" poly(MAA-co-EGDMA) microspheres (entry 1, Table 1; $270 \mathrm{mg}$ ) during their preparation, the weight of the grafted red CdTe QD-labeled polymer layer onto 
the polymer particles (i.e., the weight of the second layer of polym@rQD-polym particles) can be derived to be $270 \mathrm{mg} \times 82.5 \%=222.8 \mathrm{mg}$. Based on the above results, we could finally derive the amount of CdTe QDs in the second layer of polym@rQD-polym particles, which was obtained by dividing the weight of red CdTe QDs incorporated into polym@rQD-polym particles with the weight of the second layer on polym@rQD-polym particles (i.e., 126.2 $\mathrm{mg} / 222.8 \mathrm{mg}=0.57 \mathrm{mg} / \mathrm{mg})$.

\section{Analyses of the aqueous dispersion stability of the polymer particles}

The dispersion stability of the polymer particles in pure water was studied. After their ultrasonic dispersion in pure water $(1 \mathrm{mg} / \mathrm{mL})$, the dispersed mixtures were settled down for different time at $25{ }^{\circ} \mathrm{C}$ and their photographs were taken following the time (Figures 3, S4).

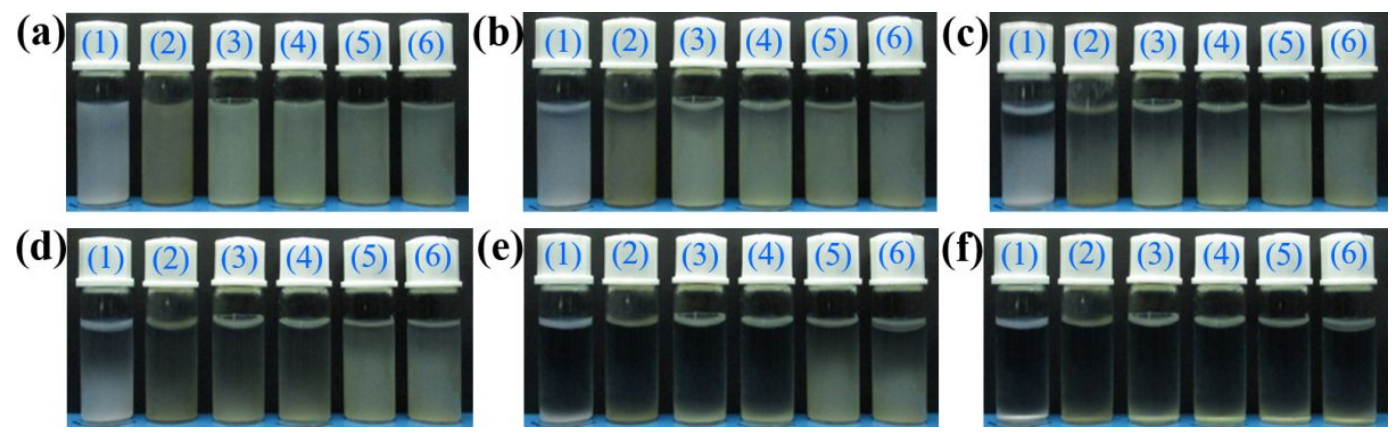

Figure S4. Dispersion photographs of poly(MAA-co-EGDMA)2 (1), polym@rQD-polym2 (2), polym@rQD-polym2@NBD-MIP(DCPAc) (3), polym@rQD-polym2@NBD-CP(DCPAc) (4),

$$
\text { polym@rQD-polym2@NBD-MIP(DCPAc)@PNIPAAm }
$$

and polym@rQD-polym2@NBD-CP(DCPAc)@PNIPAAm (6) in pure water (1 mg/mL) at $25{ }^{\circ} \mathrm{C}$ after settling down for $0 \mathrm{~h} \mathrm{(a),} 1 \mathrm{~h}$ (b), $3 \mathrm{~h}$ (c), $5 \mathrm{~h}$ (d), $7 \mathrm{~h}$ (e), and $10 \mathrm{~h}$ (f), respectively.

\section{Static water contact angle measurements}

The static water contact angles of the films prepared with polymer particles were determined as follows: The films of the polymer particles were prepared by casting their suspension solutions in DMF (10 mg/mL, after ultrasonic dispersion) on clean glass surfaces. After the solvent was evaporated at ambient temperature overnight and the films were dried under vacuum at $40{ }^{\circ} \mathrm{C}$ for 2 days, an optical contact angle analyzer (Dongguan Shengding Precision Instrument Co., Ltd., China) was used to determine their static water contact angles. Three measurements were taken across each sample, with their average being used for analyses. All the obtained results are listed in Tables 1 and S2. 
Spectrofluorimetric tirtation of red CdTe QDs and the polymerizable NBD monomer (MA-Urea-NBD) with 2,4-D (and its analogues)
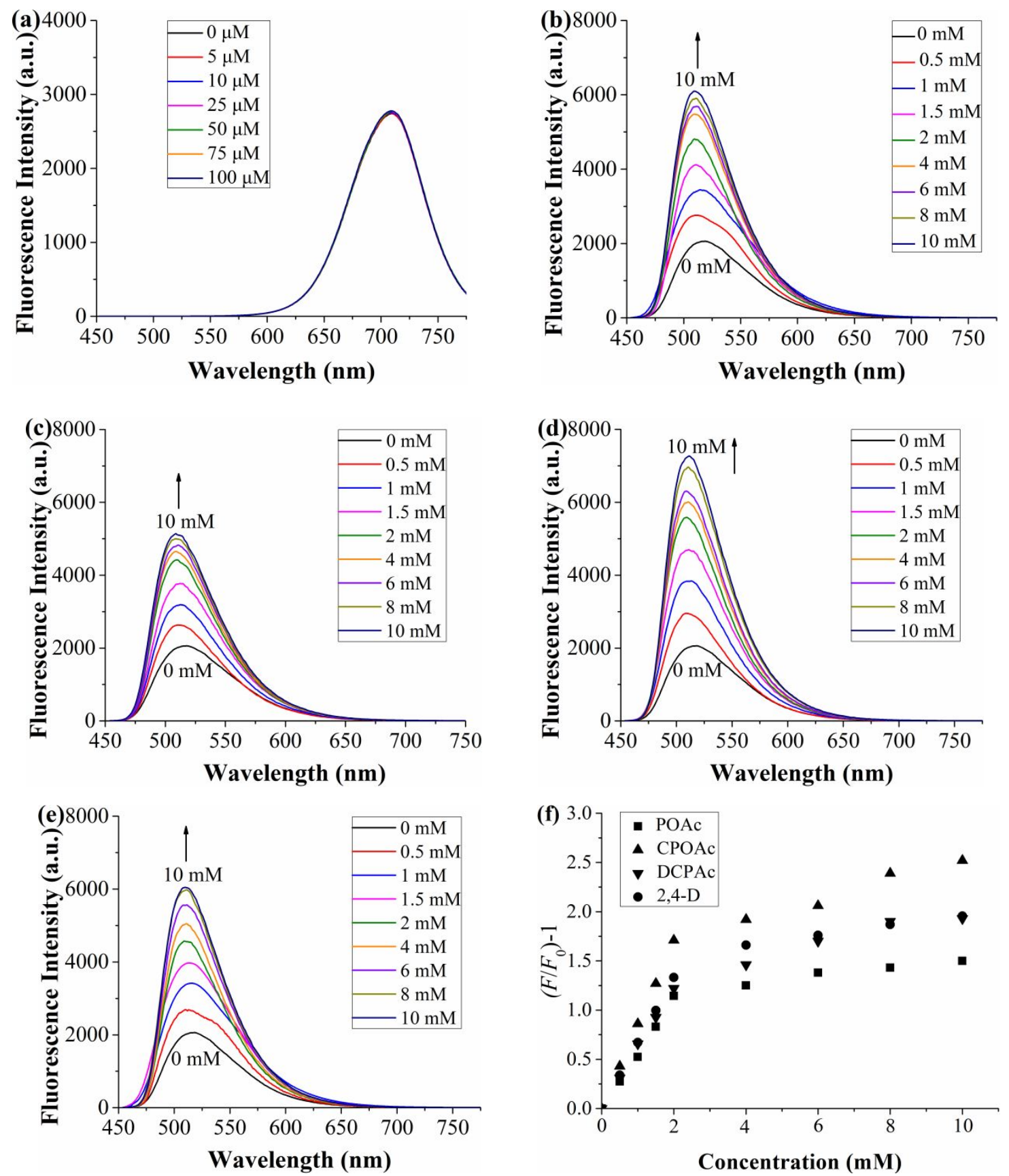

Figure S5. (a) Fluorescence spectra of the aqueous solution of red CdTe QDs $(1 \mathrm{~mL}$, as prepared) upon its exposure to different concentrations of 2,4-D at $25{ }^{\circ} \mathrm{C}$ (the incubation time is $1 \mathrm{~h})$. (b-e) Fluorescence spectra of the solutions of MA-Urea-NBD in acetonitrile $(2.5 \mathrm{mM})$ upon their exposure to different concentrations of 2,4-D (b), POAc (c), CPOAc (d), and DCPAc (e) in acetonitrile at $25{ }^{\circ} \mathrm{C}$ (the incubation time is $1 \mathrm{~h}$ ). (f) Dependence of the fluorescence enhancement of MA-Urea-NBD on the concentration of the analytes (including 2,4-D, POAc, CPOAc, and DCPAc) (derived from Figure S5b-e). 


\section{UV-vis and fluorescent spectra of the solutions of red CdTe QDs and NBD-containing fluorescent monomer}

Figure S6 presents both the UV-vis absorption spectra and fluorescence emission spectra of the solutions of red CdTe QDs and NBD-containing fluorescent monomer (MA-Urea-NBD). The UV-vis absorption spectrum of the solution of red CdTe QDs proved to have negligible overlap with the fluorescence emission spectrum of NBD, and vice versa, indicating that there exists no FRET between NBD and QDs.

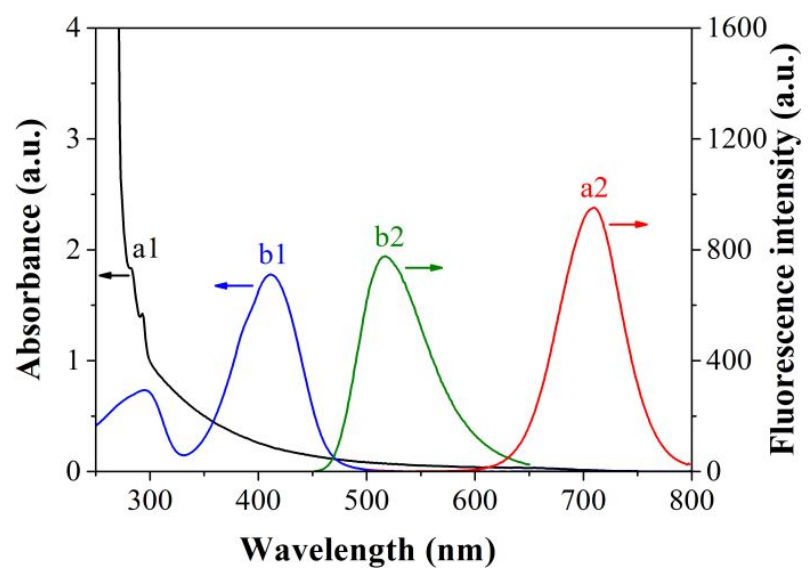

Figure S6. (a1,b1) UV-vis spectra of the solutions of CdTe QDs (surface-modified with OVDAC) in methanol (obtained by diluting the as-prepared CdTe QD solution (in methanol) 3 times with methanol) (a1) and MA-Urea-NBD in methanol (0.83 mM) (b1); (a2,b2) Fluorescent spectra of the solutions of CdTe QDs (modified with OVDAC) in methanol (obtained by diluting the as-prepared CdTe QD solution (in methanol) 3 times with methanol) (a2) and MA-Urea-NBD in methanol (0.83 mM) (b2) (excitation wavelength: $420 \mathrm{~nm})$.

\section{Confocal laser scanning microscopy (CLSM) characterization}

To confirm the full coverage of the red CdTe QD-labeled polymer layer and green NBD-labeled polymer layer on the dual fluorescent polymer microspheres, we measured the confocal laser scanning microscopy (CLSM) images of polym@rQD-polym@NBD-CP particles (entry 4 in Table 1). The CLSM images of the polymer microspheres were obtained with a NIKON A1+ confocal laser scanning microscope. All images were taken by using an oil immersion lens NA 1.45 (CFI Plan Apo Lambda 100X Oil) with a $405 \mathrm{~nm}$ solid-state laser as the excitation light. Fluorescent emission was collected at 649-679 nm and 487-601 nm, respectively, for the CLSM images of the red CdTe QD-labeled polymer layer (Figure S7a) 
and green NBD-labeled polymer layer (Figure S7b) in polym@rQD-polym@NBD-CP particles, respectively, which agrees well with the fluorescence emission wavelength range of the red CdTe QD-labeled polymer layer and green NBD-labeled polymer layer in polym@rQD-polym@NBD-CP (Figure S8). It can be seen clearly from Figure S7a,b that polym@rQD-polym@NBD-CP particles are fully covered with a red CdTe QD-labeled fluorescent layer and a green NBD-labeled fluorescent layer, respectively.
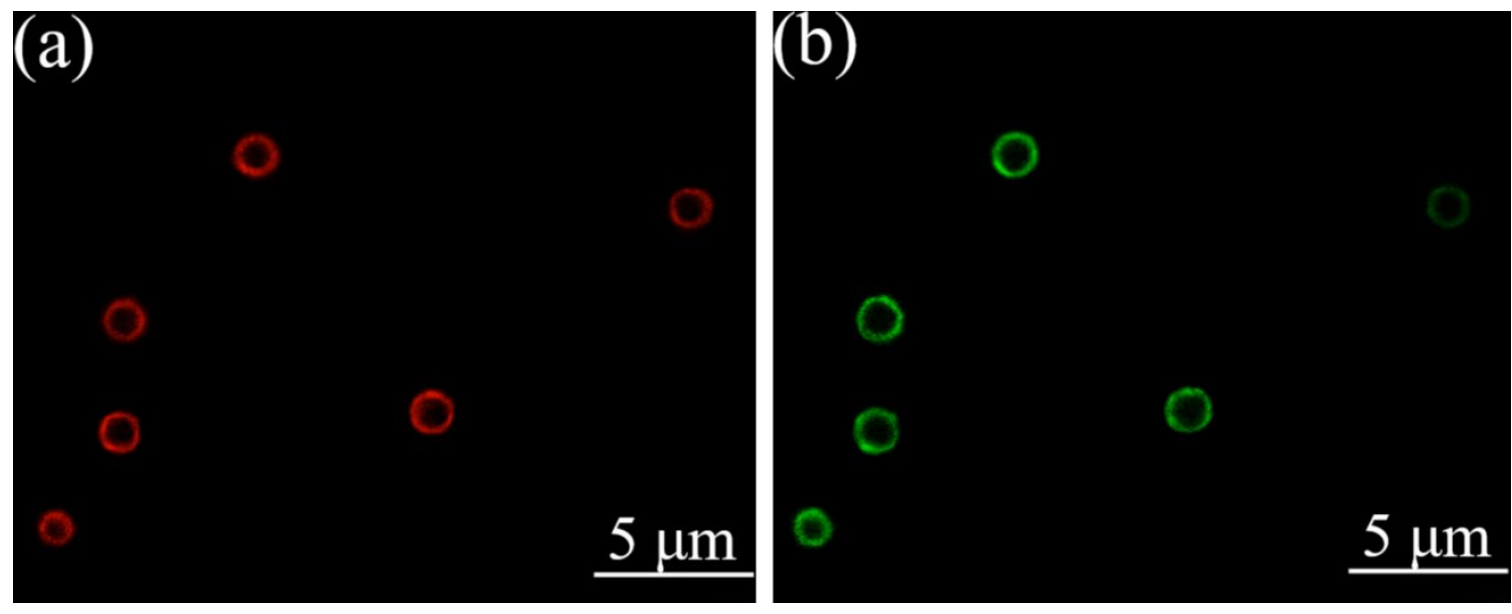

Figure S7. CLSM images of the red CdTe QD-labeled polymer layer (a) and green NBD-labeled polymer layer (b) in polym@rQD-polym@NBD-CP particles (entry 4, Table 1).

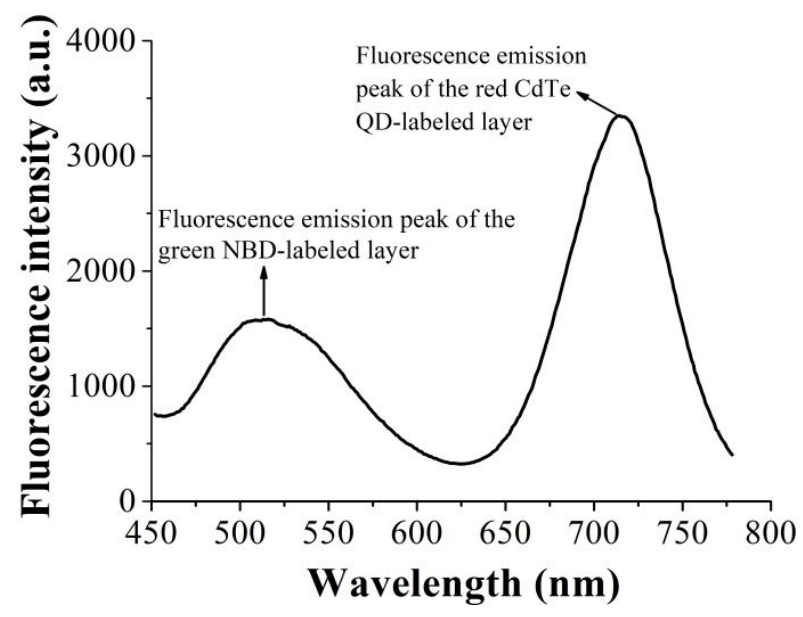

Figure S8. Fluorescent spectrum of the solution of polym@rQD-polym@NBD-CP particles (entry 4, Table 1) in acetonitrile $(0.25 \mathrm{mg} / \mathrm{mL})$ at $25^{\circ} \mathrm{C}$ (excitation wavelength: $405 \mathrm{~nm}$; voltage: $600 \mathrm{~V}$; the slit width of both the excitation and emission was $10 \mathrm{~nm}$ ).

Fluorescent properties of the ungrafted dual fluorescent 2,4-D-MIP before and after removing the template $2,4-D$ 

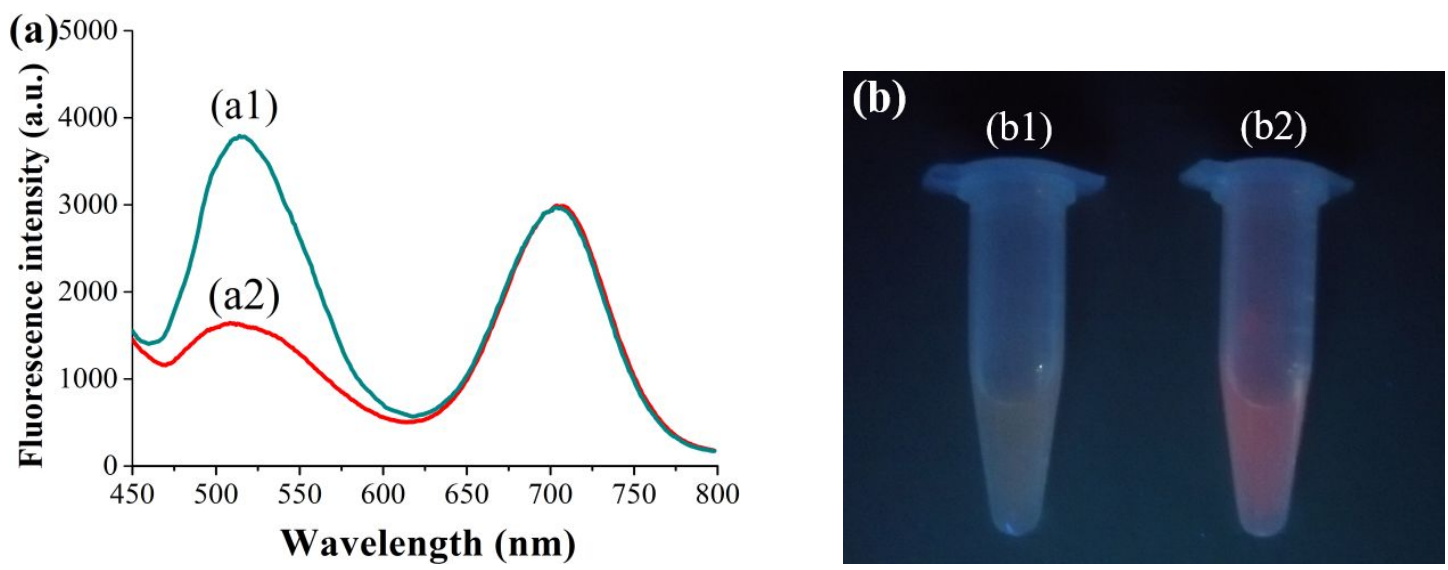

Figure S9. (a) Fluorescent spectra of the ungrafted dual fluorescent 2,4-D-MIP in acetonitrile (0.25 mg/mL) before (a1) and after (a2) removing 2,4-D; (b) Photographs of the homogeneously dispersed ungrafted dual fluorescent 2,4-D-MIP in acetonitrile $(0.25 \mathrm{mg} / \mathrm{mL})$ before (b1) and after (b2) removing 2,4-D upon exposure to $365 \mathrm{~nm}$ UV light.

\section{Equilibrium binding and competitive binding experiments with the ungrafted and grafted dual fluorescent 2,4-D-MIPs/CPs in different media}

The equilibrium binding properties of the ungrafted and grafted dual fluorescent 2,4-D-MIPs/CPs were evaluated by incubating them $(4 \mathrm{mg} / \mathrm{mL})$ with $2,4-\mathrm{D}$ in different media (including acetonitrile and undiluted pure bovine milk) $\left(C_{0}=0.02 \mathrm{mM}, 0.5 \mathrm{~mL}\right)$ at $25{ }^{\circ} \mathrm{C}$ for 8 $\mathrm{h}$, and the amounts of 2,4-D bound to MIPs/CPs (expressed as $B(\mu \mathrm{mol} / \mathrm{g})$ ) were determined by measuring those remaining in the solutions (expressed as $F$ ) with high performance liquid chromatography (HPLC, Scientific System Inc., USA) in conjunction with a UV-vis detector (i.e., $B=\left(C_{0}-F\right) V / W$, where $V$ is the volume of the used 2,4-D solution and $W$ is the mass of the used polymer). The wavelength used for the determination of 2,4-D was $284 \mathrm{~nm}$. A mixture of methanol, pure water, and acetic acid (80:19.5:0.5 v/v/v) was used as the mobile phase at a flow rate of $1 \mathrm{~mL} / \mathrm{min}$. All the above (and following) binding analyses were performed in triplicate and the mean values were used.

When the solvent of 2,4-D solutions was acetonitrile in the above binding study, the amounts of 2,4-D remaining in the supernatants (obtained after the centrifugation of the mixtures of MIPs/CPs and 2,4-D solutions) (F) were directly quantified by HPLC, from which the amounts of 2,4-D bound to MIPs/CPs could be obtained as shown above.

When the solvent of 2,4-D solutions was pure milk during the binding study, the proteins in 
the samples were removed (by adding acetonitrile into the biological samples) prior to the HPLC quantification of 2,4-D in the solutions after the template binding by MIPs/CPs following our previous method (Y. Ma, et al., Angew. Chem., Int. Ed. 2013, 52, 1511).

The selective (or competitive) binding properties of the ungrafted and grafted dual fluorescent 2,4-D-MIPs/CPs were evaluated by incubating them with a mixture of 2,4-D and its analogues (including POAc and CPOAc, Scheme S1) in different media (including acetonitrile and pure milk) at $25{ }^{\circ} \mathrm{C}$ for $8 \mathrm{~h}$, and the amounts of these analytes bound to MIPs/CPs were determined by measuring those remaining in the solutions with HPLC, following similar procedures to those shown above. The wavelength used for determining the mixed solution of 2,4-D, POAc, and CPOAc was $272 \mathrm{~nm}$. A mixture of methanol, pure water, and acetic acid (60:39.5:0.5 v/v/v) was used as the mobile phase at a flow rate of $1 \mathrm{~mL} / \mathrm{min}$.

Figure S10a,b presents the equilibrium template bindings of the ungrafted and grafted dual fluorescent 2,4-D-MIPs/CPs in acetonitrile and the undiluted pure milk, respectively.
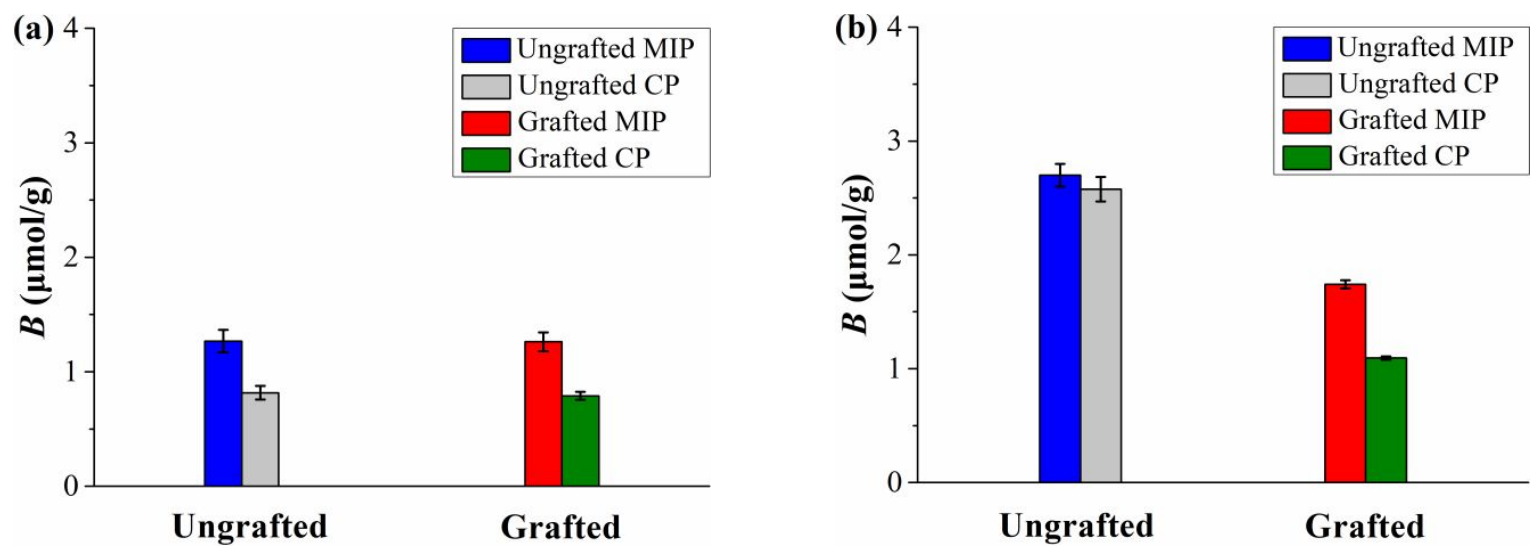

Figure S10. Equilibrium bindings of 2,4-D on the ungrafted and grafted dual fluorescent 2,4-D-MIPs/CPs in their solutions in acetonitrile (a) and the undiluted pure bovine milk (b) at $25{ }^{\circ} \mathrm{C}$, respectively $\left(C_{0(2,4-\mathrm{D})}=0.02 \mathrm{mM}\right.$; polymer concentration: $\left.4 \mathrm{mg} / \mathrm{mL}\right)$.

Figure S11a,b presents the competitive bindings of the ungrafted and grafted dual fluorescent 2,4-D-MIPs/CPs toward 2,4-D and its structural analogues POAc and CPOAc in acetonitrile and pure milk, respectively (note that DCPAc was not used here because we could not separate it from 2,4-D, POAc, and CPOAc under the studied HPLC elution condition), from which the "imprinting-induced promotion of binding" (IPB) values of the ungrafted and grafted dual fluorescent 2,4-D-MIPs were derived and listed in Table S3. 

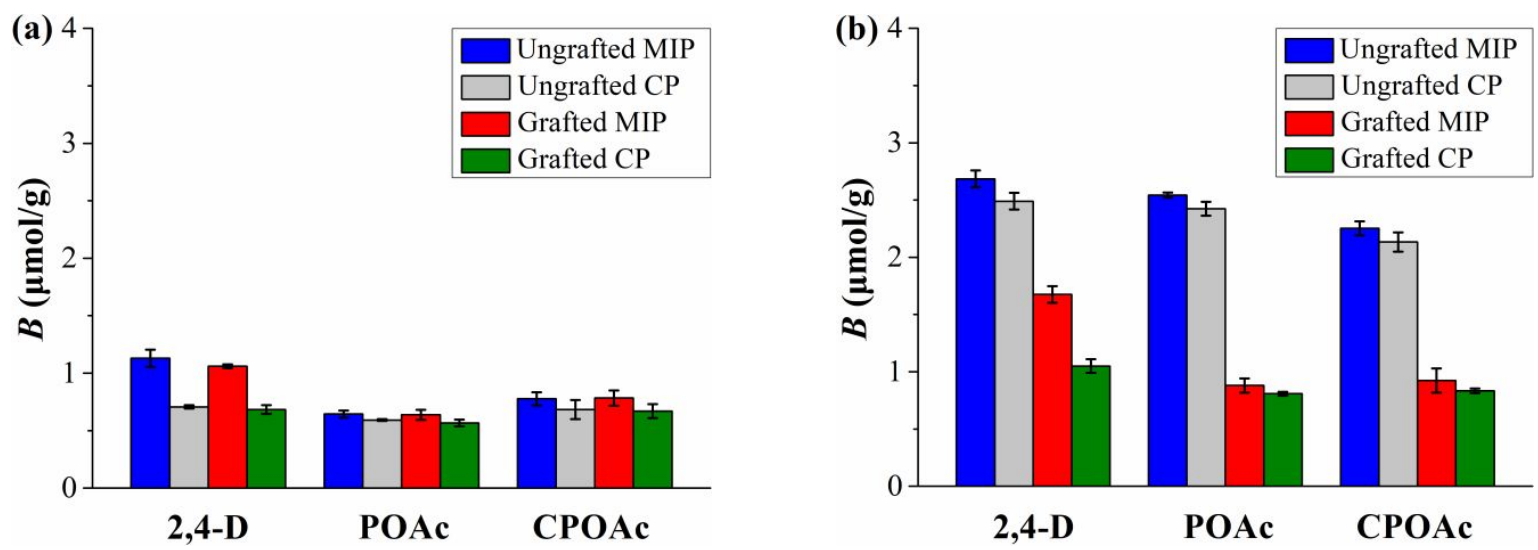

Figure S11. Selective bindings of the ungrafted and grafted dual fluorescent 2,4-D-MIPs/CPs toward 2,4-D, POAc, and CPOAc in their mixed solutions in acetonitrile (a) and the undiluted pure bovine milk (b), respectively $\left(C_{0(2,4-\mathrm{D} \text { or POAC or } \mathrm{CPOAc})}=0.02 \mathrm{mM}\right.$; polymer concentration: $4 \mathrm{mg} / \mathrm{mL})$.

Table S3. Selective binding properties of the ungrafted and grafted dual fluorescent 2,4-D-MIPs/CPs toward 2,4-D and its analogues in different media.

\begin{tabular}{|c|c|c|c|c|c|c|c|}
\hline \multirow{2}{*}{ Solvent } & \multirow{2}{*}{ Analyte } & \multicolumn{3}{|c|}{$\begin{array}{l}\text { The ungrafted dual fluorescent } \\
\text { 2,4-D-MIP/CP }\end{array}$} & \multicolumn{3}{|c|}{$\begin{array}{c}\text { The grafted dual fluorescent } \\
\text { 2,4-D-MIP/CP }\end{array}$} \\
\hline & & $B_{\mathrm{MIP}}{ }^{a}$ & $B_{\mathrm{CP}}^{a}$ & IPB $(\%)^{b}$ & $B_{\mathrm{MIP}}{ }^{a}$ & $B_{\mathrm{CP}}{ }^{a}$ & IPB $(\%)^{b}$ \\
\hline \multirow{3}{*}{ Acetonitrile } & $2,4-\mathrm{D}$ & $1.13 \pm 0.08$ & $0.71 \pm 0.02$ & 59 & $1.06 \pm 0.02$ & $0.68 \pm 0.04$ & 56 \\
\hline & POAc & $0.65 \pm 0.03$ & $0.59 \pm 0.01$ & 10 & $0.64 \pm 0.04$ & $0.57 \pm 0.03$ & 12 \\
\hline & CPOAc & $0.78 \pm 0.06$ & $0.68 \pm 0.08$ & 15 & $0.78 \pm 0.07$ & $0.67 \pm 0.06$ & 16 \\
\hline \multirow{3}{*}{ Pure milk } & 2,4-D & $2.69 \pm 0.07$ & $2.49 \pm 0.07$ & 8 & $1.68 \pm 0.07$ & $1.05 \pm 0.06$ & 60 \\
\hline & POAc & $2.54 \pm 0.02$ & $2.42 \pm 0.06$ & 5 & $0.88 \pm 0.06$ & $0.81 \pm 0.02$ & 9 \\
\hline & CPOAc & $2.25 \pm 0.06$ & $2.13 \pm 0.09$ & 6 & $0.92 \pm 0.11$ & $0.83 \pm 0.02$ & 11 \\
\hline
\end{tabular}

${ }^{a} B_{\mathrm{MIP}}$ and $B_{\mathrm{CP}}$ are the equilibrium binding capacities of the studied MIP and its CP toward $2,4-\mathrm{D}$ and its analogues in their mixed solution $\left(C_{2,4-\mathrm{D} \text { or POAc or } \mathrm{CPOAc}}=0.02 \mathrm{mM}\right)$ in different media, which are the same as those shown in Figure S11a,b and have a unit of $\mu \mathrm{mol} / \mathrm{g} ;{ }^{b}$ IPB refers to the "imprinting-induced promotion of binding" value of the studied MIP, IPB $(\%)=$ $\left[\left(B_{\mathrm{MIP}}-B_{\mathrm{CP}}\right) / B_{\mathrm{CP}}\right] \times 100$.

Binding isotherms of the grafted dual fluorescent 2,4-D-MIP/CP in the undiluted pure bovine milk

The study on the binding isotherms of the grafted dual fluorescent 2,4-D-MIP/CP was carried out as follows: MIP/CP particles (2 mg) were incubated with a series of 2,4-D solutions in the undiluted pure bovine milk $(C=0.005 \sim 0.1 \mathrm{mM}, 0.5 \mathrm{~mL})$ at $25{ }^{\circ} \mathrm{C}$ for $8 \mathrm{~h}$. After centrifugation, the amounts of the template remaining in the supernatants $(F)$ were quantified by HPLC following our previous method (Y. Ma, et al., Angew. Chem. Int. Ed. 2013, 52, 
$1511)$, from which the amounts of 2,4-D bound to the MIP/CP $(B=C-F)$ were derived.

Figure $\mathrm{S} 12 \mathrm{a}$ presents the template binding isotherms (in the format of $B$ versus $F$ ) of the grafted dual fluorescent 2,4-D-MIP/CP in the undiluted pure bovine milk. The grafted MIP proved to bind more template than its corresponding $\mathrm{CP}$ in a wide range of 2,4-D concentrations, again indicating the presence of imprinted binding sites in the studied MIP.
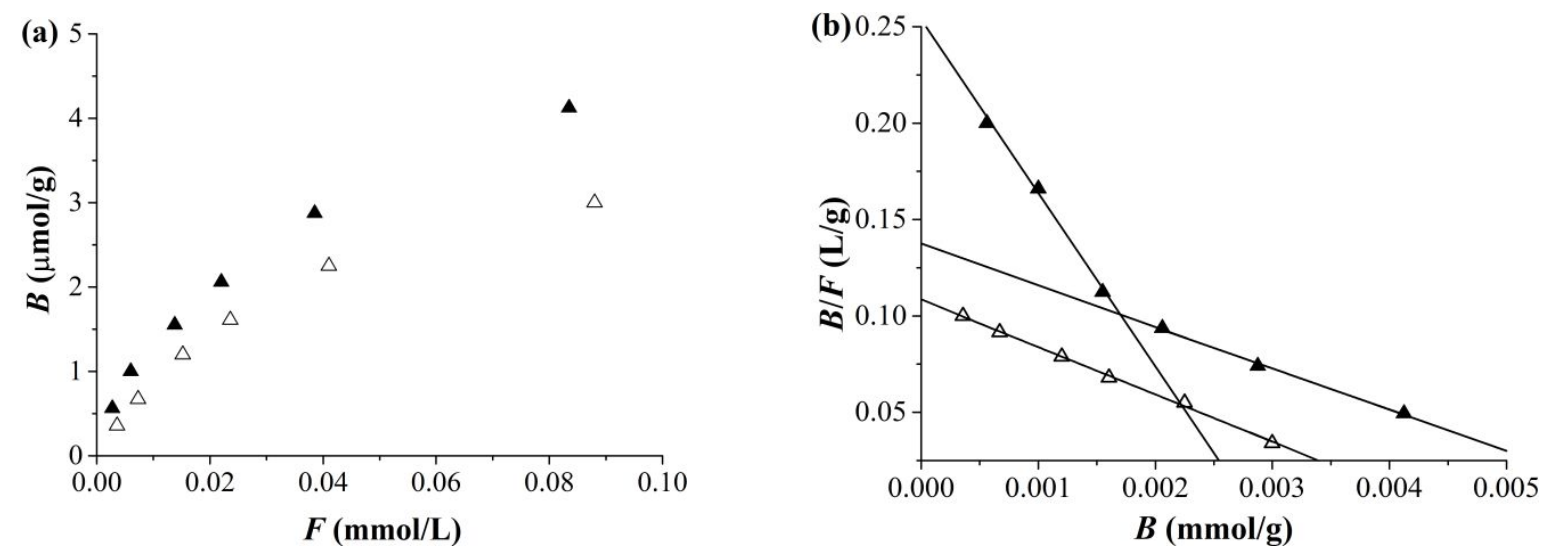

Figure S12. (a) Binding isotherms (in the format of $B$ versus $F$ ) of the grafted dual fluorescent 2,4-D-MIP (filled symbol)/CP (open symbol) in pure milk; (b) Scatchard plots of the grafted dual fluorescent 2,4-D-MIP (filled symbol)/CP (open symbol) in pure milk.

Scatchard analysis has proven to be a versatile approach for obtaining binding parameters of the MIPs (R. J. Umpleby II, et al., J. Chromatogr. B 2004, 804, 141). The Scatchard equation used is $B / F=\left(N_{\max }-B\right) K_{\mathrm{a}}$, where $K_{\mathrm{a}}$ and $N_{\max }$ represent the binding association constant and apparent maximum number of the binding sites, respectively. The binding isotherms of the grafted dual fluorescent 2,4-D-MIP/CP in the undiluted pure bovine milk were thus plotted in the format of $B / F$ versus $B$ (Figure $\mathrm{S} 12 \mathrm{~b}$ ). The Scatchard plot of the grafted dual fluorescent 2,4-D-MIP proved to be fitted into two straight lines, suggesting that the binding sites in the studied MIP are heterogeneous and their affinities can be approximated by two binding association constants $\left(K_{\mathrm{a}}\right)$ corresponding to the high- and low-affinity sites, as usually observed for the MIPs prepared via the noncovalent molecular imprinting approach (Y. Watabe, et al., J. Chromatogr. A 2005, 1073, 363). As expected, the Scatchard plot of the corresponding CP could only be fitted into one straight line, suggesting their only presence of homogeneous and nonspecific binding sites on their surfaces. The binding parameters (including $K_{\mathrm{a}}$ and $N_{\max }$ values) of the grafted dual fluorescent 2,4-D-MIP/CP in the undiluted pure bovine milk are summarized in Table S4. 
Table S4. The binding parameters of the grafted dual fluorescent 2,4-D-MIP/CP in pure milk obtained from Scatchard analysis

\begin{tabular}{|c|c|c|c|c|c|c|}
\hline \multirow{3}{*}{ Solvent } & \multicolumn{4}{|c|}{ Grafted dual fluorescent 2,4-D-MIP } & \multirow{2}{*}{\multicolumn{2}{|c|}{$\begin{array}{l}\text { Grafted dual } \\
\text { fluorescent 2,4-D-CP }\end{array}$}} \\
\hline & \multicolumn{2}{|c|}{ High-affinity sites } & \multicolumn{2}{|c|}{ Low-affinity sites } & & \\
\hline & $K_{\mathrm{a}}\left(\mathrm{M}^{-1}\right) \times 10^{-4}$ & $N_{\max }(\mu \mathrm{mol} / \mathrm{g})$ & $K_{\mathrm{a}}\left(\mathrm{M}^{-1}\right) \times 10^{-4}$ & $N_{\max }(\mu \mathrm{mol} / \mathrm{g})$ & $K_{\mathrm{a}}\left(\mathrm{M}^{-1}\right) \times 10^{-4}$ & $N_{\max }(\mu \mathrm{mol} / \mathrm{g})$ \\
\hline Pure milk & 8.9 & 2.8 & 2.1 & 6.5 & 2.5 & 4.3 \\
\hline
\end{tabular}

Optosensing properties of the grafted (and ungrafted) dual fluorescent 2,4-D-MIP/CP and DCPAc-MIP/CP in the undiluted pure bovine milk

\section{Photostability measurements of the grafted dual fluorescent 2,4-D-MIP/CP}

The photostability of the grafted dual fluorescent 2,4-D-MIP/CP was determined by measuring the fluorescence intensity changes of their dispersed mixtures in pure water $(0.25 \mathrm{mg} / \mathrm{mL})$ over time at $25^{\circ} \mathrm{C}$ under air atmosphere (Figures $4 \mathrm{a}$ and S13).

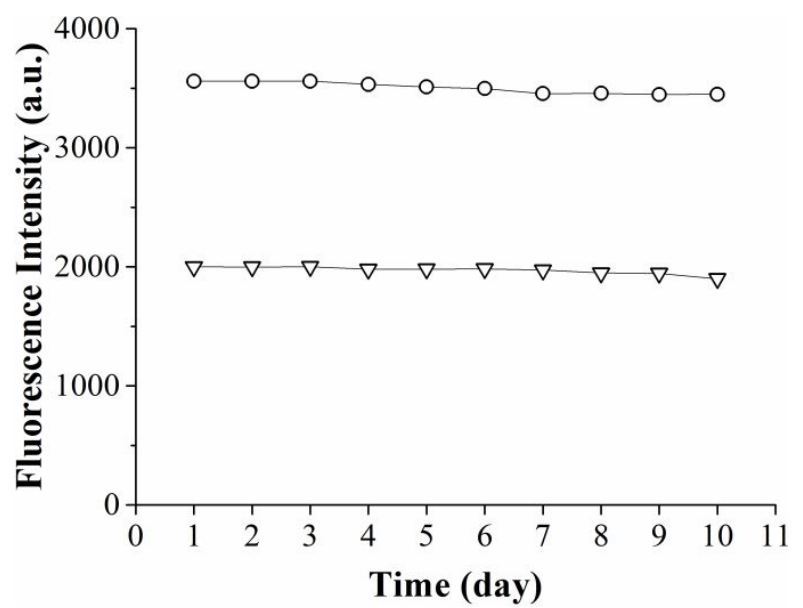

Figure S13. Fluorescence intensity changes of a dispersed mixture of the grafted dual fluorescent 2,4-D-CP in pure water $(0.25 \mathrm{mg} / \mathrm{mL})$ (including the red CdTe QDs (circle) and green NBD fluorophores (triangle) embedded inside CP particles) over time at $25^{\circ} \mathrm{C}$ under air atmosphere.

\section{Reusability of the grafted dual fluorescent 2,4-D-MIP/CP}

The reusability of the grafted dual fluorescent 2,4-D-MIP/CP was assessed by measuring the fluorescence intensity changes of their dispersed mixtures in the undiluted pure bovine milk $(0.25 \mathrm{mg} / \mathrm{mL})$ after their consecutive 2,4-D adsorption-desorption cycles as follows: $0.25 \mathrm{mg}$ of the studied 2,4-D-MIP or 2,4-D-CP was first dispersed in $1 \mathrm{~mL}$ of pure milk, and the fluorescence intensities of the mixture were determined. After centrifugation of the above 
mixture and removal of the supernatant, the resulting MIP or CP was washed with pure water thrice and then with methanol twice, followed by their drying with argon flowing. The dried MIP or CP was then incubated with a 2,4-D solution in pure milk $(15 \mu \mathrm{M}, 1 \mathrm{~mL})$ at $25{ }^{\circ} \mathrm{C}$ for 2 $\mathrm{h}$, and the fluorescence intensities of this mixture were determined. Afterwards, the above mixture was centrifuged again, the resulting MIP or CP with bound 2,4-D was first washed with pure water thrice and then thoroughly with methanol until no 2,4-D could be detectable with HPLC in the washing solution. The resulting MIP or CP was then dried with argon flowing. The dried MIP or CP was dispersed in $1 \mathrm{~mL}$ of pure milk and the fluorescence intensities of the above mixture were again determined. The above procedure was repeated for 10 cycles and the results are presented in Figures $4 \mathrm{~b}$ and S14.

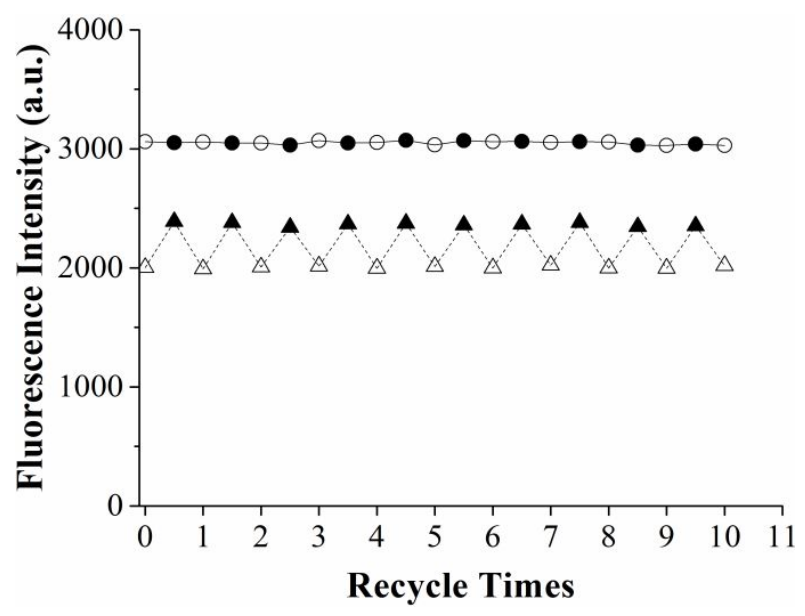

Figure S14. Fluorescence intensity changes of the grafted dual fluorescent 2,4-D-CP during its 10 regeneration cycles (for the red CdTe QDs (circle) and green NBD fluorophores (triangle) embedded inside CP particles $(0.25 \mathrm{mg} / \mathrm{mL})$, respectively, upon desorption (empty) and adsorption (filled) of 2,4-D (15 $\mu \mathrm{M})$ in the undiluted pure bovine milk).

Study on the binding kinetics of the grafted dual fluorescent 2,4-D-MIP/CP and DCPAC-MIP/CP

The binding kinetics of the grafted dual fluorescent 2,4-D-MIP/CP were obtained by incubating them with a 2,4-D solution in the undiluted pure milk and examining their fluorescence change over time (Figures 5 and S15). 


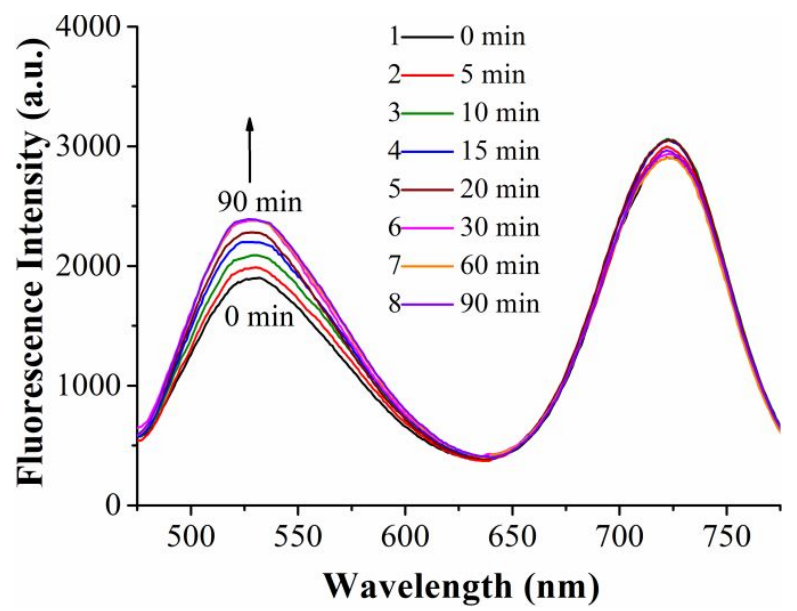

Figure S15. Fluorescence spectra of the grafted dual fluorescent 2,4-D-CP $(0.25 \mathrm{mg} / \mathrm{mL})$ after its incubation with a 2,4-D solution $(15 \mu \mathrm{M})$ in the undiluted pure bovine milk at $25{ }^{\circ} \mathrm{C}$ for different time.

Similarly, the binding kinetics of the grafted dual fluorescent DCPAc-MIP/CP were also obtained by incubating them with a DCPAc solution in the undiluted pure milk and examining their fluorescence change over time (Figure S16).
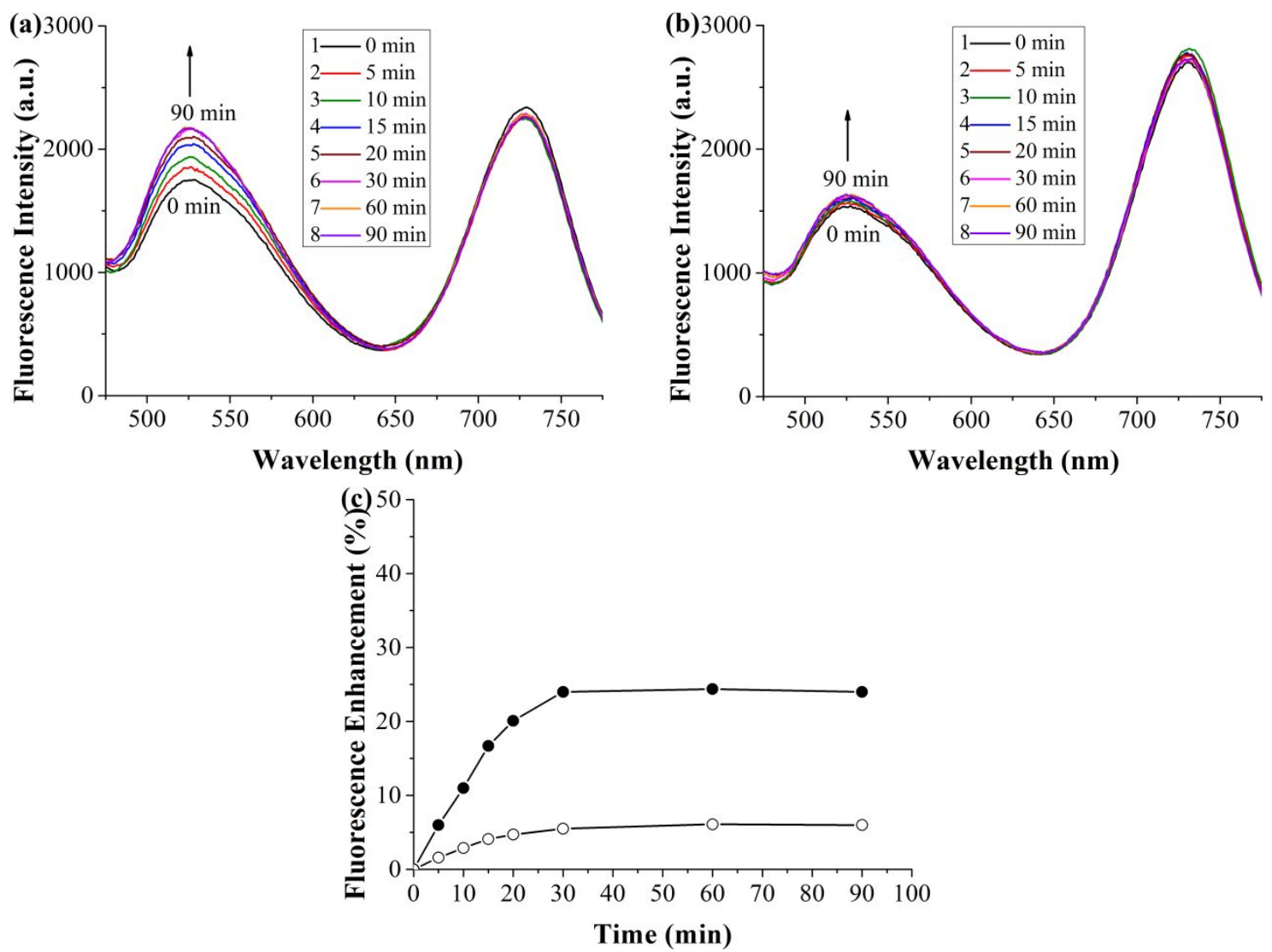

Figure S16. $(a, b)$ Fluorescence spectra of the grafted dual fluorescent DCPAc-MIP (a) and DCPAc-CP (b) $(0.25 \mathrm{mg} / \mathrm{mL})$ after their incubation with a DCPAc solution $(15 \mu \mathrm{M})$ in the 
undiluted pure bovine milk at $25{ }^{\circ} \mathrm{C}$ for different time. (c) Binding kinetics of the grafted dual fluorescent DCPAc-MIP (filled symbol)/CP (open symbol) $(0.25 \mathrm{mg} / \mathrm{mL}$ ) in a DCPAc solution $(15 \mu \mathrm{M})$ in the undiluted pure milk at $25{ }^{\circ} \mathrm{C}$ (derived from Figures $\mathrm{S} 16 \mathrm{a}$ and $\mathrm{b}$; Fluorescence enhancement $\left.(\%)=\left[\left(F_{t}-F_{0}\right) / F_{0}\right)\right] \times 100$, where $F_{t}$ and $F_{0}$ are the fluorescence intensity of the green NBD unit (at $525 \mathrm{~nm}$ ) at a time of $t$ and 0 , respectively).

Spectrofluorimetric tirtation of the ungrafted dual fluorescent 2,4-D-MIP/CP with 2,4-D in the undiluted pure bovine milk
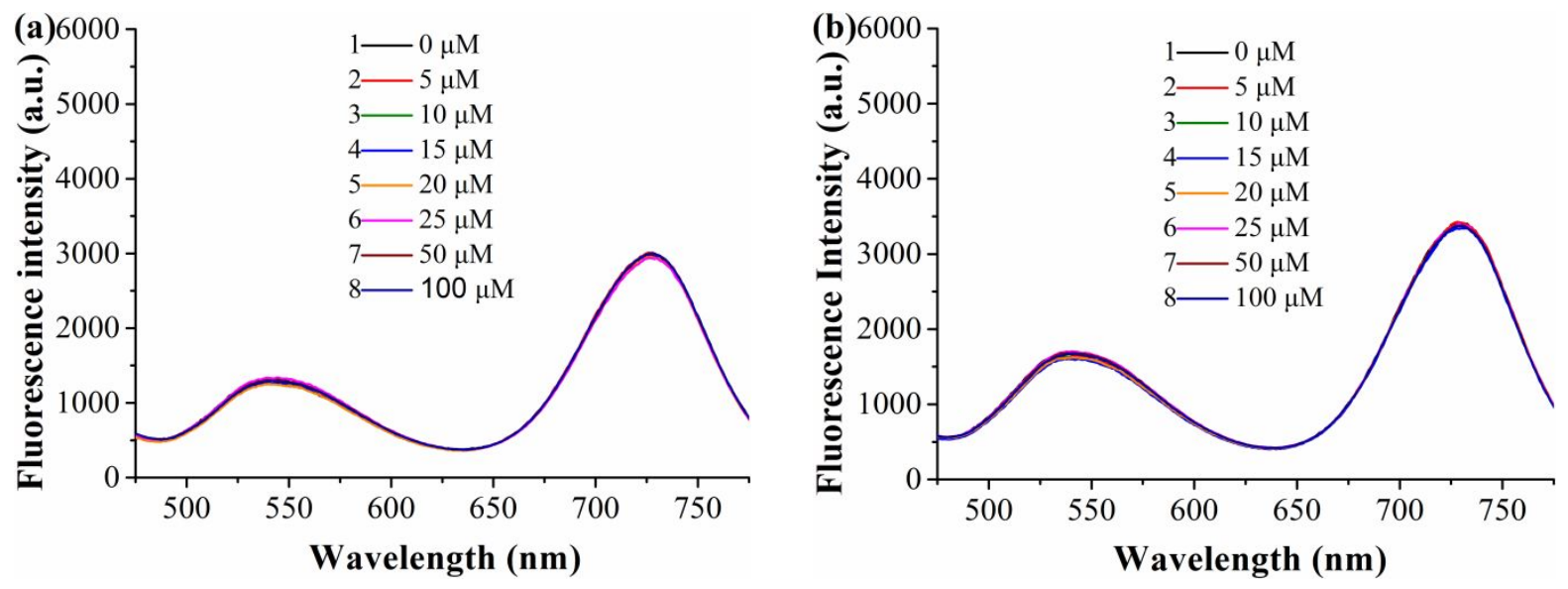

Figure S17. Fluorescence spectra of the ungrafted dual fluorescent 2,4-D-MIP (a) and 2,4-D-CP (b) $(0.25 \mathrm{mg} / \mathrm{mL})$ upon their incubating with different concentrations of 2,4-D in the undiluted pure milk at $25^{\circ} \mathrm{C}$ for $2 \mathrm{~h}$.

Spectrofluorimetric tirtation of the grafted dual fluorescent DCPAc-MIP/CP with DCPAc in the undiluted pure bovine milk
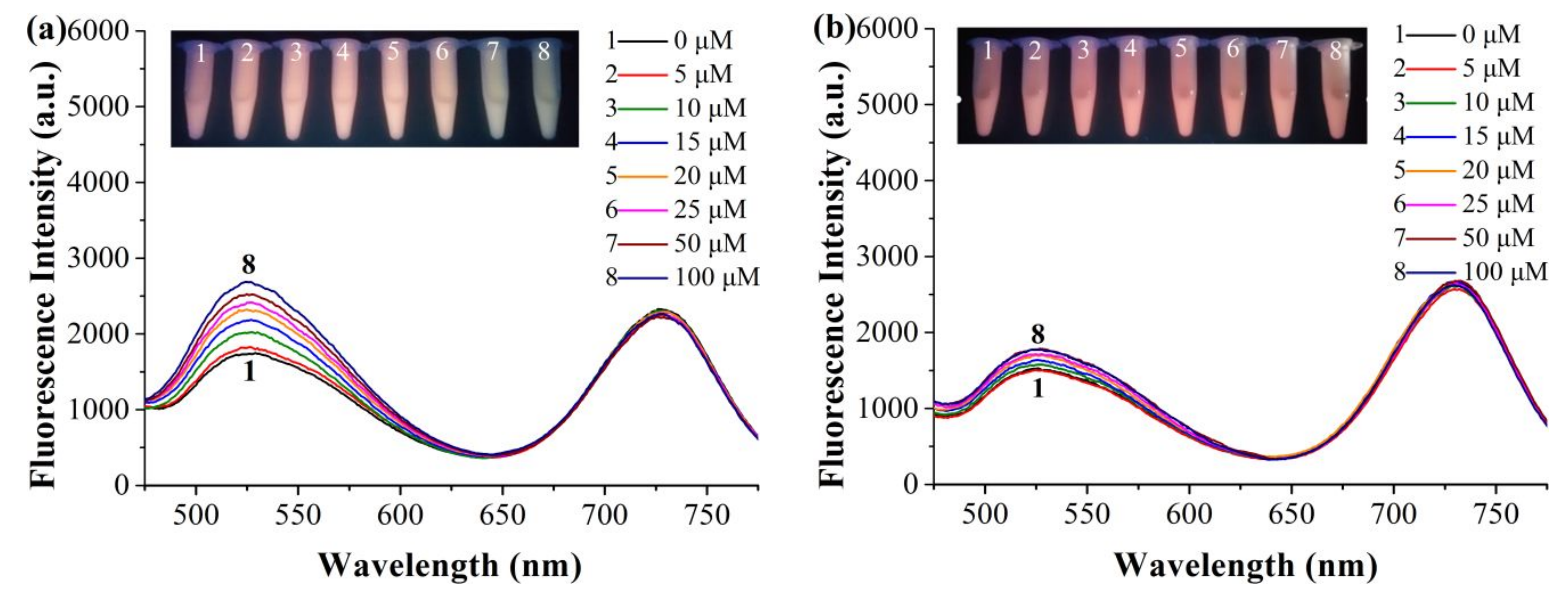


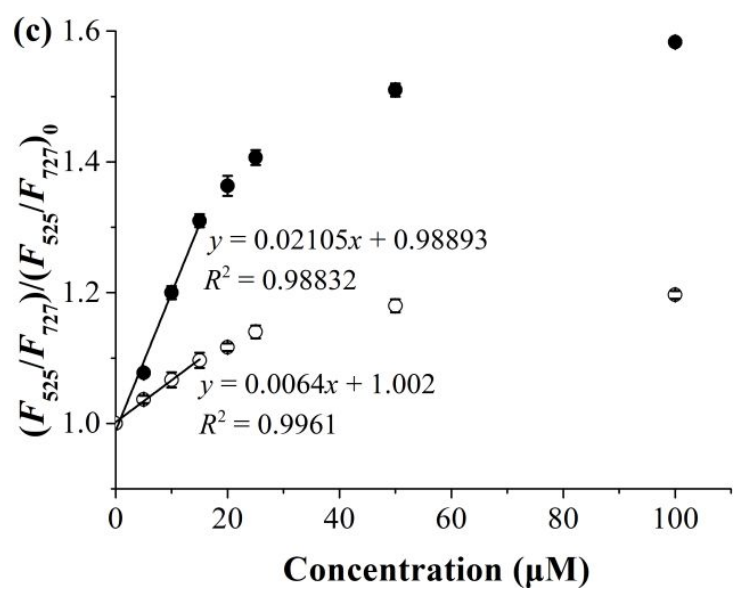

Figure S18. Fluorescence spectra of the grafted dual fluorescent DCPAc-MIP (a)/CP (b) (0.25 $\mathrm{mg} / \mathrm{mL}$ ) upon their incubating with different concentrations of DCPAc in the undiluted pure bovine milk at $25{ }^{\circ} \mathrm{C}$ for $2 \mathrm{~h}$ (Inset: fluorescence color changes of the MIP/CP upon their exposure to different concentrations of DCPAc). (c) Dependence of the fluorescence enhancement of the grafted dual fluorescent DCPAc-MIP (filled symbol)/CP (open symbol) on the DCPAc concentration (derived from Figure S18a,b).

Selective optosensing properties of the grafted dual fluorescent DCPAc-MIP/CP in the undiluted pure bovine milk
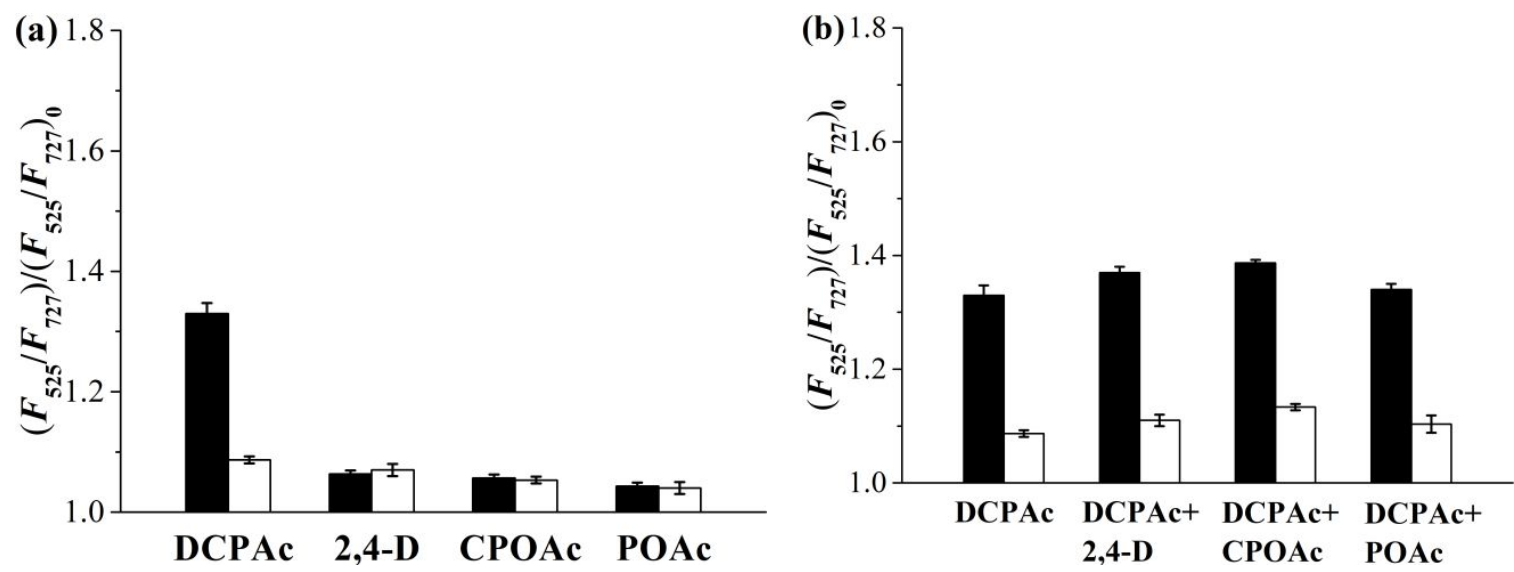

Figure S19. Fluorescence enhancement of the grafted dual fluorescent DCPAc-MIP (filled column)/DCPAc-CP (open column) $(0.25 \mathrm{mg} / \mathrm{mL})$ after their incubation with a solution of DCPAc, 2,4-D, CPOAc, or POAc $\left(C_{\mathrm{DCPAc}}\right.$,2,-D, CPOAc, or POAc $\left.=15 \mu \mathrm{M}\right)(\mathrm{a})$ or with a solution of DCPAc $(15 \mu \mathrm{M})$ in the presence of the same concentration of 2,4-D, CPOAc, or POAc (b) in the undiluted pure bovine milk at $25^{\circ} \mathrm{C}$ for $2 \mathrm{~h}$. 
Direct quantification of DCPAc in the undiluted pure goat milk with the grafted dual fluorescent DCPAc-MIP

Table S5. Application of the grafted dual fluorescent DCPAc-MIP for direct quantification of DCPAc in the undiluted pure goat milk spiked with different amounts of DCPAc or mixtures of DCPAc and some interfering substances.

\begin{tabular}{|c|c|c|c|c|}
\hline \multirow[t]{2}{*}{ Entry } & \multirow[t]{2}{*}{ Anayte(s) } & \multicolumn{2}{|c|}{ Concentration of analyte $(\mathrm{s})(\mu \mathrm{M})$} & \multirow{2}{*}{$\begin{array}{l}\text { Recovery } \pm \text { RSD } \\
(\%)(n=3)\end{array}$} \\
\hline & & Spiked & Found (DCPAc) & \\
\hline 1 & DCPAc & 0.5 & $0.49 \pm 0.01$ & $97.3 \pm 2.3$ \\
\hline 2 & DCPAc & 5 & $5.15 \pm 0.12$ & $103 \pm 2.4$ \\
\hline 3 & DCPAc & 10 & $9.73 \pm 0.05$ & $97.3 \pm 0.5$ \\
\hline 4 & $\begin{array}{l}\mathrm{DCPAc}+2,4-\mathrm{D}+ \\
\mathrm{CPOAc}+\mathrm{POAc}\end{array}$ & $\begin{array}{c}0.5(\mathrm{DCPAc})+0.5(2,4-\mathrm{D})+ \\
0.5(\mathrm{CPOAc})+0.5(\mathrm{POAc})\end{array}$ & $0.5 \pm 0.02$ & $100.3 \pm 4.5$ \\
\hline 5 & $\begin{array}{l}\mathrm{DCPAc}+2,4-\mathrm{D}+ \\
\mathrm{CPOAc}+\mathrm{POAc}\end{array}$ & $\begin{array}{c}5(\mathrm{DCPAc})+5(2,4-\mathrm{D})+ \\
5(\mathrm{CPOAc})+5(\mathrm{POAc})\end{array}$ & $5.24 \pm 0.03$ & $104.9 \pm 0.5$ \\
\hline 6 & $\begin{array}{l}\mathrm{DCPAc}+2,4-\mathrm{D}+ \\
\mathrm{CPOAc}+\mathrm{POAc}\end{array}$ & $\begin{array}{c}10(\text { DCPAc })+10(2,4-D)+ \\
10(\text { CPOAc })+10(\text { POAc })\end{array}$ & $10.19 \pm 0.19$ & $101.9 \pm 1.9$ \\
\hline
\end{tabular}

